\title{
Atomic layer deposited second-order nonlinear optical metamaterial for back-end integration with CMOS-compatible nanophotonic circuitry
}

\author{
Stéphane Clemmen ${ }^{1,2,+}$, Artur Hermans ${ }^{1,2,+}$, Eduardo Solano ${ }^{3}$, Jolien \\ Dendooven ${ }^{3}$, Kalle Koskinen ${ }^{4}$, Martti KaURanen ${ }^{4}$, Edouard Brainis ${ }^{2,5}$, \\ Christophe DetaVernier ${ }^{3}$, Roel Baets ${ }^{1,2}$
}

\author{
1. Photonics Research Group, Department of Information Technology, Ghent University-imec, \\ Sint-Pietersnieuwstraat 41, 9000 Ghent, Belgium \\ 2. Center for Nano- and Biophotonics (NB-Photonics), Ghent University, Sint-Pietersnieuwstraat 41, 9000 Ghent, Belgium \\ 3. Department of Solid State Sciences, Faculty of Sciences, Ghent University, Krijgslaan 281/S1, 9000 Ghent, Belgium \\ 4. Optics Laboratory, Tampere University of Technology, PO Box 692, Fl-33101 Tampere, Finland \\ 5. Physics and Chemistry of Nanostructures Group, Ghent University, Krijgslaan 281/S3, 9000 Ghent, Belgium \\ + Author e-mail address: sclemmen@intec.ugent.be; these authors contributed equally to the work
}

Received XX Month XXXX; revised XX Month, XXXX; accepted XX Month XXXX; posted XX Month XXXX (Doc. ID XXXXX); published XX Month XXXX

\begin{abstract}
We report the fabrication of artificial unidimensional crystals exhibiting an effective bulk second-order nonlinearity. The crystals are created by cycling atomic layer deposition of three dielectric materials such that the resulting metamaterial is non-centrosymmetric in the direction of the deposition. Characterization of the structures by second-harmonic generation Maker-fringe measurements shows that the main component of their nonlinear susceptibility tensor is about $5 \mathrm{pm} / \mathrm{V}$ which is comparable to well-established materials and more than an order of magnitude greater than reported for a similar crystal [L. Alloatti et al., Appl. Phys. Lett. 107, 121903 (2015)]. Our demonstration opens new possibilities for second-order nonlinear effects on CMOS-compatible nanophotonic platforms.
\end{abstract}

OCIS codes: (190.4400) Nonlinear optics, materials, (190.4720), Optical nonlinearities of condensed matter, (190.4350) Nonlinear optics at surfaces

http://dx.doi.org

Second-order nonlinear optical response of materials gives rise to useful effects, including nonlinear wave mixing and the Pockels effect, with applications such as light generation in optical parametric oscillators and electro-optic modulation. Some of these applications have been miniaturized using various technologies. More recently, a goal has been to integrate optical functionalities on nanophotonic chips that are compatible with CMOS fabrication, which is the standard in micro/nanoelectronics. As a result, optical parametric oscillators [1,2] and fast modulators [3] have been reported. Since silicon and silicon nitride, which are the two main CMOS-compatible photonics platforms, lack a second-order nonlinearity, those realizations were based on the third-order nonlinearity or carrier effects. This resulted in a modest improvement in terms of energy consumption and efficiency over simpler second-order nonlinear devices widely used in free space nonlinear optics. Therefore, it would be highly desirable to be able to induce a second-order nonlinear response in a material otherwise lacking that property.
To date, common methods to artificially create a second-order nonlinearity include poling in silica glass [4] or polymers [5], strain [6], plasmonic surface enhancement [7], and alternate stacking of organic films [8]. In addition, even materials expected to lack a second-order response may in some cases exhibit a significant response, but the origin remains unknown $[9,10]$. In any case, the inversion symmetry of the material structure must somehow be broken to induce a second-order nonlinear response.

In this letter, we utilize the symmetry breaking mechanism which was also implemented by Alloatti et al. in 2015 [11] to induce a substantial second-order nonlinear response, as described by the second-order susceptibility $\chi^{(2)}$. We deposit very thin layers of three distinct transparent amorphous materials $\mathrm{A}, \mathrm{B}$, and $\mathrm{C}$ and repeat that structure many times to form a thick layer of a composite $\mathrm{ABC}$ material. In such a system, each interface between any two materials breaks the symmetry resulting in an effective bulk $\chi^{(2)}$ for the overall structure. Whereas [11] reported a relatively low second-order response, we demonstrate that such an $\mathrm{ABC}$ approach can result in a large $\chi^{(2)}$, comparable to that of well-known second-order materials. Our characterization is based on second-harmonic generation (SHG) Maker-fringe measurements that allow the nonlinearity of the $\mathrm{ABC}$ layer to be separated unambiguously from that of the substrate. We verified that the SHG contributions of each of the 3 interfaces A-B, B-C, and $C$-A do not sum up to 0 as would be expected for an $A B$ system.

It is important to understand that our approach is well suited for integration with existing CMOS-compatible nanophotonics platforms. Indeed, the deposition method, ALD, is conformal, requires low temperature, and has been proven to integrate perfectly with existing nanophotonic circuitry [12]. Moreover, as the symmetry of the ABC structure is broken along its normal, the nonlinearity requires electric field components along the normal direction, which occurs for ppolarized light at non-normal incidence (see figure 1a). This implies that, for the case of widely used planar or rib waveguides, the nonlinearity would be the greatest for a TM-mode such as illustrated in figure $1 b$.

In the present proof of principle, the three materials were chosen to be (A) $\mathrm{TiO}_{2}$, (B) $\mathrm{Al}_{2} \mathrm{O}_{3}$, and (C) $\mathrm{In}_{2} \mathrm{O}_{3}$. While detailed theories exist to predict second-order nonlinearities of superlattices of crystalline materials [13], we are not aware of such theories existing for stacks of amorphous materials. In choosing our particular materials, we therefore used 


\begin{tabular}{|c|c|c|c|c|c|}
\hline 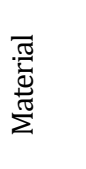 & 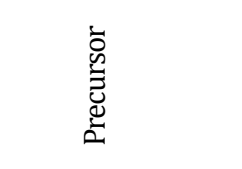 & 专离 & 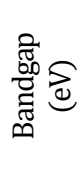 & 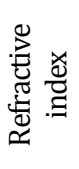 & 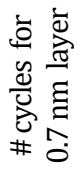 \\
\hline $\mathrm{TiO}_{2}$ & $\begin{array}{l}\text { TDMAT - Tetrakis } \\
\text { (dimethylamido)ti } \\
\text { tanium }\end{array}$ & 0.06 & $\begin{array}{c}3.4 \\
{[15]}\end{array}$ & $\begin{array}{c}2.1 \\
{[16]}\end{array}$ & 12 \\
\hline $\mathrm{Al}_{2} \mathrm{O}_{3}$ & $\begin{array}{c}\text { TMA- } \\
\text { Trimethylaluminiu } \\
\text { m }\end{array}$ & 0.1 & $\begin{array}{c}8.8 \\
{[17]}\end{array}$ & $\begin{array}{c}1.5- \\
1.7 \\
{[18]}\end{array}$ & 7 \\
\hline $\mathrm{In}_{2} \mathrm{O}_{3}$ & $\begin{array}{l}\text { In(TMHD)3- } \\
\text { Tris(2,2,6,6- } \\
\text { tetramethyl-3,5- } \\
\text { heptanedionato) } \\
\text { indium(III) }\end{array}$ & 0.01 & $\begin{array}{c}3.7 \\
{[19]}\end{array}$ & $\begin{array}{c}2.2 \\
{[19]}\end{array}$ & 70 \\
\hline
\end{tabular}

Table 1: Summary of ALD parameters and optical properties. For the TDMAT and In(TMHD) 3 precursors, Ar was used as a carrier gas.

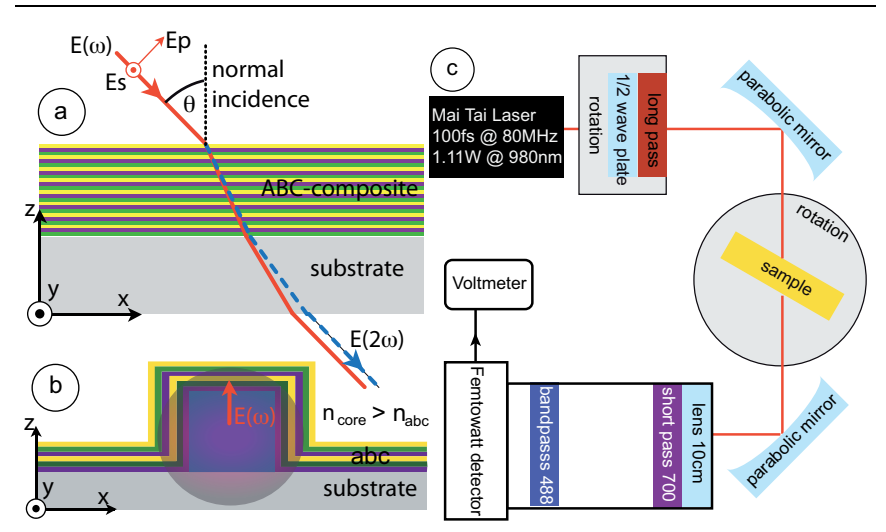

Figure 1: (a) Geometry of the ABC composite and the incidence of either $\mathrm{p}$ - or s-polarized light resulting in collinear second-harmonic generation. (b) Possible use of the nonlinearity via the TM-mode of a waveguiding structure (c) Maker-fringe experimental setup for characterization of $\chi^{(2)}$ nonlinearity via second-harmonic generation.

\begin{tabular}{|c|c|}
\hline \multicolumn{2}{|c|}{ ABC composite } \\
\hline $\begin{array}{c}\mathrm{n}_{\mathrm{z}}(980 \mathrm{~nm}) \\
\text { ordinary }\end{array}$ & 2.02 \\
\hline $\begin{array}{c}\mathrm{n}_{\mathrm{z}}(490 \mathrm{~nm}) \\
\text { ordinary }\end{array}$ & 2.13 \\
\hline $\begin{array}{c}\mathrm{n}_{\mathrm{x}}(980 \mathrm{~nm}) \\
\text { extraordinary }\end{array}$ & 1.92 \\
\hline $\begin{array}{c}\mathrm{n}_{\mathrm{x}}(490 \mathrm{~nm}) \\
\text { extraordinary }\end{array}$ & 2.06 \\
\hline
\end{tabular}

\begin{tabular}{|c|c|}
\hline \multicolumn{2}{|c|}{ Borosilicate glass } \\
\hline $\mathrm{n}(980 \mathrm{~nm})$ & 1.4633 \\
\hline $\mathrm{N}(490 \mathrm{~nm})$ & 1.4766 \\
& \\
\hline$\chi_{\text {xxz }}^{\text {glass }}\left(\mathrm{m}^{2} / \mathrm{V}\right)$ & $\begin{array}{c}11.6 \pm 0.8 \\
\times 10^{-22}[27]\end{array}$ \\
\hline$\chi_{\text {zzz }}^{\text {glass }}\left(\mathrm{m}^{2} / \mathrm{V}\right)$ & $93 \pm 14$ \\
$\times 10^{-22}[27]$ \\
\hline
\end{tabular}

Table 2: Parameters used for the fitting (see body of the text for more details) Left: Refractive indices of the $\mathrm{ABC}$ composite measured by ellipsometry at the fundamental and second-harmonic wavelengths for ordinary and extraordinary polarization. Right: refractive index and nonlinearity of the borosilicate glass substrate.

the old guideline that the nonlinearity depends on the dielectric contrast between the materials [14] (see table 1). In consequence, the nonlinearities of the A-B and B-A interfaces are equal in magnitude but opposite in sign. By combining the three materials appropriately, however, we expect to obtain a structure where the nonlinearities of the A-B, B-C, and C-A interface have a non-cancelling contribution. Each individual layer is $0.7 \mathrm{~nm}$ thick such that the $\mathrm{ABC}$ cycle is repeated many times to form thick $A B C$ composite layers on top of $500 \mu \mathrm{m}$ hick borosilicate glass substrates (Schott BOROFLOAT®33). The thickness of individual layers is an arbitrary compromise between the greatest density of interfaces and the certainty to have a well-defined layer. Several ABC composite crystals were made that differ only by their total thickness of 2.1, 25 and $50 \mathrm{~nm}$ which is limited solely by the duration of the deposition process. The deposition process started with the cleaning of the glass with $\mathrm{O}_{2}$ plasma. The ALD was enhanced by using an oxygen plasma with an RF power of $200 \mathrm{~W}$, a frequency of $13.56 \mathrm{MHz}$ and a pulse duration of $10 \mathrm{~s}$. The deposition was done by alternating pulses of the corresponding metalorganic precursor at a pressure of $6.0 \times 10^{-5}$ bar, followed by the $0_{2}$ plasma pulse at $1.2 \times 10^{-5}$ bar and a temperature of $120^{\circ} \mathrm{C}$, constant throughout the full deposition process. In between each gas pulse the chamber is pumped down to high vacuum. Table 1 shows the precursors and growth-percycle for each of the three materials deposited [20-23].

Since the individual layers are much thinner than optical wavelengths, we can consider the multilayer as one homogeneous uniaxial material [24]. The refractive indices of the $\mathrm{ABC}$ composite were measured via ellipsometry (see table 2).

The nonlinear characterization was done using the Maker-fringe technique [25] with the setup depicted in figure 1c. The source for fundamental light was a commercial Ti:sapphire laser (Mai Tai HP from Spectra-Physics) emitting $100 \mathrm{fs}$ pulses at the fundamental wavelength of $980 \mathrm{~nm}$ and a peak power reaching $140 \mathrm{~kW}$. The linear polarization of the laser beam was aligned to p-polarization with a halfwave plate and its spectrum was cleaned to suppress any spurious light at wavelengths below $800 \mathrm{~nm}$. Then, the light is slightly focused on the sample using a parabolic mirror of $5 \mathrm{~cm}$ focal length such that the beam size $\left(1 / \mathrm{e}^{2}\right)$ is brought from $1.2 \mathrm{~mm}$ to $52 \pm 4$ microns. The corresponding Rayleigh range is $9 \mathrm{~mm}$ thus leaving a relatively large tolerance for the alignment of the sample in the focal plane. After interaction with the sample, the light was collimated and the fundamental wavelength filtered out from the generated secondharmonic light. A lens was placed before the femtowatt detector (Thorlabs PDF10A) so that it can accommodate beam displacements induced by the rotation of the sample. To confirm that the detected signal is SHG and not any fluorescence, we tuned the laser wavelength so that its corresponding second-harmonic wavelength falls out of the bandpass filter and verified that the signal collected vanishes.

The results of our experiment consist of Maker fringes for our samples consisting of a blank substrate, and substrates coated on one side by 2.1, 25, and $50 \mathrm{~nm}$ of our $\mathrm{ABC}$ composite material. The results are summarized in figures 2 and 3 . The curve corresponding to the bare borosilicate glass shows the expected Maker fringes with a visibility limited in part by temporal walk-off occurring between the fundamental beam and the second-harmonic signal generated at the first interface and for the rest due to experimental imperfection leading to slightly unequal intensities at the front and back surfaces. The curve corresponding to $2.1 \mathrm{~nm}$ sample shows essentially the same response as the blank substrate thus providing a first indication that the air-ABC interface is not responsible for the increased SHG. The curves corresponding to thicker ABC samples show SHG increasing with thickness and fringes with reduced visibility. The reduced visibility is due to imbalance between the increased responses of the $\mathrm{ABC}$ layers and the unchanged response of the uncoated back surface.

To extract a value for the second-order susceptibility from these measurements, we model and fit the experimental curves assuming that SHG occurs at the ABC layer at the front interface and at the glassair back interface. The SHG depends on the respective components of the second-order nonlinear susceptibility tensor $\chi_{x x z}^{a b c}, \chi_{z x x}^{a b c}, \chi_{z Z z}^{a b c}$ of the ABC composite and $\chi_{x x z}^{\text {glass }}, \chi_{z x x}^{\text {glass }}, \chi_{z z z}^{\text {glass }}$ of the glass substrate. The SHG signals can be described by the following equation [26]:

$$
\begin{aligned}
& E_{\text {total }}=E_{\text {front }}+E_{\text {back }} \\
& \propto\left[\frac{t_{\text {air }, a b c}^{2} T_{a b c, \text { glass }} T_{\text {glass }, \text { air }}}{N_{a b c} \cos \left(\Theta_{\text {abc }}\right)} \exp \left(i \frac{2 L \omega N_{\text {glass }} \cos \Theta_{\text {glass }}}{c}\right) \chi^{a b c}\right. \\
& \left.+\frac{t_{\text {air }, a b c}^{2} t_{\text {abc,glass }}^{2} T_{\text {glass }, a i r}}{N_{\text {glass }} \cos \left(\Theta_{\text {glass }}\right)} \exp \left(i \frac{2 L \omega n_{\text {glass }} \cos \theta_{\text {glass }}}{c}\right) \chi^{\text {glass }}\right]
\end{aligned}
$$


with

and

$$
\begin{aligned}
\chi^{a b c}=\chi_{x x z}^{a b c} \sin (2 & \left.\theta_{a b c}\right) \cos \left(\Theta_{a b c}\right) \\
& +\chi_{z x x}^{a b c} \sin \left(\Theta_{a b c}\right) \cos ^{2}\left(\theta_{a b c}\right) \\
& +\chi_{z z z}^{a b c} \sin ^{2}\left(\theta_{a b c}\right) \sin \left(\Theta_{a b c}\right)
\end{aligned}
$$

$$
\begin{aligned}
\chi^{\text {glass }}=\chi_{x x z}^{\text {glass }} \sin & \left(2 \theta_{\text {glass }}\right) \cos \left(\Theta_{\text {glass }}\right) \\
& +\chi_{\text {zxx }}^{\text {glass }} \sin \left(\Theta_{\text {glass }}\right) \cos ^{2}\left(\theta_{\text {glass }}\right) \\
& +\chi_{\text {zzz }}^{\text {glass }} \sin ^{2}\left(\theta_{\text {glass }}\right) \sin \left(\Theta_{\text {glass }}\right)
\end{aligned}
$$

The generated fields depend on parameters defined at the fundamental frequency $\omega$ (lower case letters) and second-harmonic frequency $2 \omega$ (capital letters), such as the Fresnel transmission coefficients $t_{i, j}$ and $T_{i j}$ at each interface, the propagation angles $\theta$ and $\Theta$ of the beams in the ABC layer and in the glass, as well as the refractive indices of the glass substrate $n_{\text {glass }}$ and $\mathrm{N}_{\text {glass. }}$. As our model does not account for the birefringence of the material, we also neglected it in the Fresnel transmission coefficients and set the indices as the average of the ordinary and extraordinary indices ( $\mathrm{n}=1.97, \mathrm{~N}=2.1$ ). The thickness $L$ and dispersion of the glass substrate are responsible for the period of the Maker fringes as the intensity is given by $\mathrm{I}=\mathrm{c}_{1}\left|\mathrm{E}_{\text {front }}+\mathrm{E}_{\text {back }}\right|^{2}$ where $\mathrm{c}_{1}$ is a proportionality constant. The fitting procedure contains three real-valued free parameters: $c_{1}$, $\chi_{\mathrm{zzz}}^{\mathrm{abc}}$ and $\left(2 \chi_{\mathrm{xxz}}^{\mathrm{abc}}+\chi_{\mathrm{zxx}}^{\mathrm{abc}}\right)$. Other parameters used for the fitting are summarized in table 2. Assuming that the SHG originates from an effective bulk nonlinearity of the $\mathrm{ABC}$ layer, the respective bulk secondorder susceptibility is obtained by dividing the measured surface-type signals by the thickness $\mathrm{D}$ of the $\mathrm{ABC}$ layer so that $\chi_{\mathrm{zzz}} \equiv \frac{\chi_{\mathrm{zz}}^{\mathrm{abc}}}{D}$ and $A_{z x} \equiv \frac{\left(2 \chi_{x \times z}^{\mathrm{abc}}+\chi_{z \times x}^{\mathrm{abc}}\right)}{D}$. The small discrepancy between experimental and fitting curve at small angles of incidence may be due to multiple reflections between the front and back surfaces, which our model does not account for. Our characterization also suffers from imperfections that manifest themselves in the nondiagonal components $\left(2 \chi_{\mathrm{xxz}}^{\mathrm{abc}}+\chi_{\mathrm{zxx}}^{\mathrm{abc}}\right)$., whose values for different samples vary by a factor of two. Note, however, that these components are particularly sensitive to the quality of the fit for small angles of incidence, whereas the diagonal component is relatively more important for large angles of incidence, where the fit is very good.

From this fitting procedure, we deduce the nonlinearity to be $\chi_{\mathrm{zzz}}=6.1 \pm 0.4 \mathrm{pm} / \mathrm{V} \quad\left(\chi_{\mathrm{zzz}}=6.0 \pm 0.8 \mathrm{pm} / \mathrm{V}\right) \quad$ and $A_{z x}=0.78 \pm 0.07 \mathrm{pm} / \mathrm{V}\left(A_{z x}=1.44 \pm 0.16 \mathrm{pm} / \mathrm{V}\right)$ for the $50 \mathrm{~nm}$ $(25 \mathrm{~nm})$ thick samples. Note that we find similar values for the bulk nonlinearities of the $50 \mathrm{~nm}$ and $25 \mathrm{~nm}$ thick samples. This shows that the SHG indeed originates from the bulk of the ABC layer rather than the ABC-air interface. Moreover it indicates that our measurements were not influenced by impurities adsorbed on the samples' surfaces which could alter the surface nonlinearities. Clearly, the main diagonal tensor component $\chi_{\mathrm{zzz}}$ is significantly larger than the value of $0.26 \mathrm{pm} / \mathrm{V}$ reported before for a similar nanocomposite [11]. Assuming that Kleinman symmetry is satisfied, we can impose $\chi_{\mathrm{xxz}}=\chi_{\mathrm{zzx}}$ and then deduce also an order of magnitude value for $\chi_{\mathrm{zzx}} \approx 0.35 \pm 0.15 \mathrm{pm} / \mathrm{V}$.

To gain further insight on the relative strengths between the diagonal and non-diagonal components, we also measured SHG from our $50 \mathrm{~nm}$ thick sample as a function of the polarization of the fundamental beam at a fixed incidence angle. Figure 3 shows that the SHG signal vanishes almost perfectly for s-polarized incident light, which confirms that the non-diagonal components of the nonlinear tensor are indeed much weaker than the diagonal one. Further studies are needed to obtain more precise values for the nondiagonal tensor components of our $\mathrm{ABC}$ composite.

To further demonstrate that the origin of the effective bulk nonlinearity is the broken centrosymmetry resulting from the $\mathrm{ABC}$ structure, we have acquired additional Maker fringes (see figure 4) from a structure where two $\mathrm{ABC}$ samples were brought together.
The reference curve (crosses) corresponds to SHG from two blank glass substrates thus showing a weak response. The level of SHG is almost identical to the reference curve presented in figure 2 but presents fringes with a shorter period and reduced visibility as a consequence of the doubled thickness of the substrate. In particular, the temporal walk-off becomes more significant because of the thicker sample. The second curve (squares) is obtained using two identical ABC samples, coated on one side of the substrate. The two

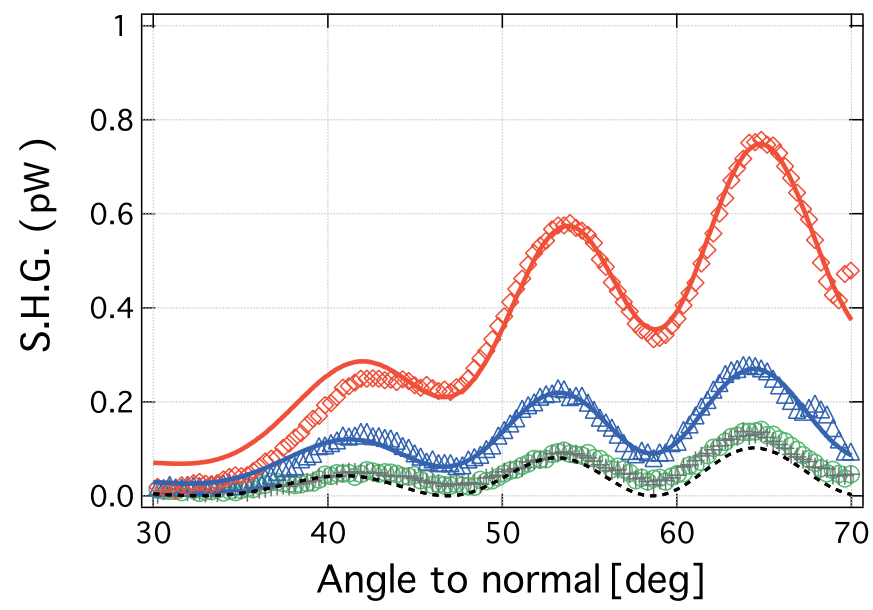

Figure 2: Experimental data (markers) and fitting curves (bold lines) of the second harmonic generation (average power) as a function of the incidence angle for $50 \mathrm{~nm}$ (diamonds), $25 \mathrm{~nm}$ (triangles), $2.1 \mathrm{~nm}$ (circles) and $0 \mathrm{~nm}$ (crosses) thick ABC composite coating deposited on the front surface of a Borofloat wafer.

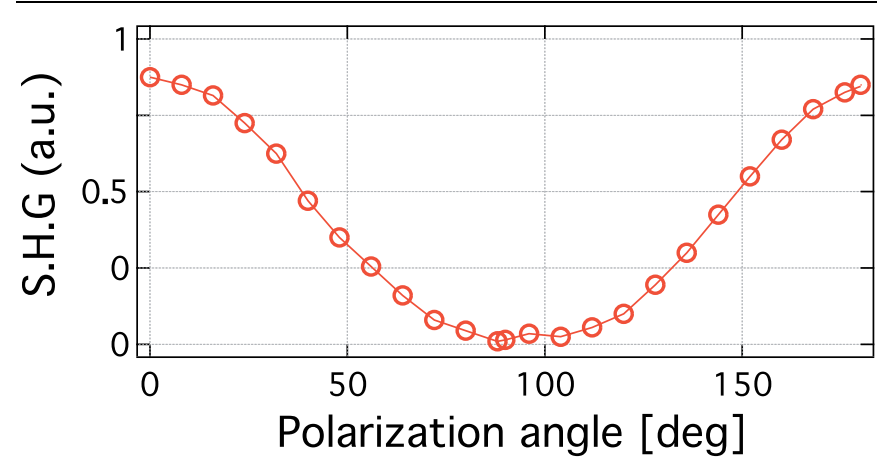

Figure 3: Variation of the SHG with the incident polarization angle.

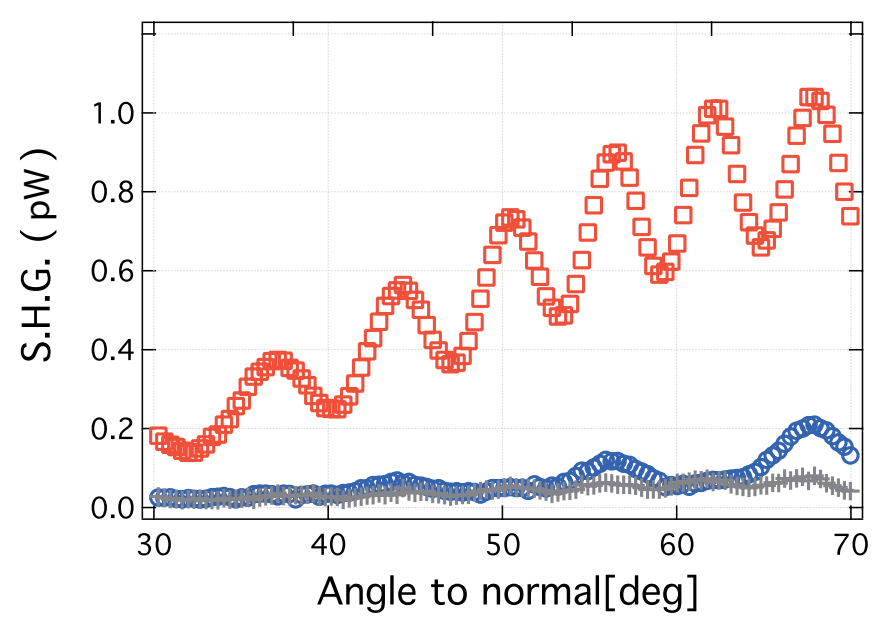

Figure 4: Comparison of SHG (average power) originating from 2 partsamples: ABC-glass+glass-CBA (red squares), glass-CBA+ABC-glass (blue circles), glass+glass (grey crosses). 
samples face each other on their uncoated sides so that the interfaces producing SHG are the two ABC layers while the glass-glass interface does not produce SHG. This results in strong SHG with fringes exhibiting similar visibility as the blank sample. However, the visibility is still reduced for the reasons mentioned above. Finally, the last curve (circles) corresponds to those two same ABC-coated substrates but facing each other on their coated side. The nonlinear material has thus a restored symmetry as its cycling structure is now ABC...ABC-CBA...CBA and it is expected to result in negligible SHG. Indeed, while the SHG contributions from the air-glass interfaces of course remain, the curves indicate that the contributions from the $\mathrm{ABC}$ layers vanish to a large extent.

We should note that the samples of the present study were far from being optimized. In addition, our experimental setup was not yet optimized for the most precise measurements. While this affects mainly the weaker non-diagonal tensor components, both the diagonal and non-diagonal components are partly coupled through our fitting procedure. In order to take this remaining uncertainty into account, we believe that it is safe to state that the value of the dominant component is $\chi_{\mathrm{zzz}}$ is $5 \pm 2 \mathrm{pm} / \mathrm{V}$.

Nevertheless this value for the main tensor component is greater by more than an order of magnitude than the $0.26 \mathrm{pm} / \mathrm{V}$ reported by Alloatti et al. [11] for a similar system. We note that only one material is different in the $\mathrm{ABC}$ composite in [11]: $\mathrm{HfO}_{2}$ is used where we have $\mathrm{In}_{2} \mathrm{O}_{3}$. Since $\chi^{(2)}$ is expected to be proportional to the density of interfaces, we also assess this quantity in both cases. Each $A B C$ period is $2.7 \mathrm{~nm}$ in [11] as compared to $2.1 \mathrm{~nm}$ in our study, so we find it can only explain for a difference of a factor 1.3 for the secondorder susceptibility. This seems to indicate that the materials chosen, and likely also the deposition parameters, have a considerable influence on the effective bulk second-order susceptibility. Finally we want to mention that in [11] the surface nonlinearity of the glass substrate, and thus the interference between SHG from the front and back surfaces, is not accounted for; despite the fact that the Rayleigh range is much thicker than the sample. This can result in an underestimation of the nonlinearity when operating in a Makerfringe minimum. However, it cannot accommodate for an order of magnitude difference. We think an important future study would be to investigate the possible correlation between the linear and/or nonlinear susceptibilities of many combinations of $\mathrm{ABC}$ materials and the resulting effective second-order susceptibility.

In conclusion, we have demonstrated an artificial nonlinear material relying on the principle of surface induced symmetry breaking. We believe that this new class of nonlinear material is promising as our proof of principle indicates a second-order nonlinearity reaching $5 \pm 2 \mathrm{pm} / \mathrm{V}$ for its main tensor component. We believe that such a second-order nonlinearity could be used in combination with nanophotonic waveguides based on CMOS-compatible materials that lack significant second-order nonlinearity. We envisage sub-micron SiN waveguides with guided modes overlapping over $50 \%$ with the $\mathrm{ABC}$ composite coated on top of the waveguide. Furthermore, the possibilities to increase the nonlinearity of the $\mathrm{ABC}$ composite are numerous ranging from thinner individual layers to optimization of the contrast between the materials involved [14].

Funding. S.C., R.B., A.H., and J.D. would like to thank the ERC-InSpectra advanced grant and FWO-Vlaanderen for funding support. K.K. and M.K. acknowledge Tampere University of Technology for Optics and Photonics Strategic Funding. K.K. acknowledges the Vaisala Foundation for a Fellowship.

Acknowledgment. We thank Koen Alexander for his support in the automatization of the experimental setup.

\section{References}

1. J. S. Levy, A. Gondarenko, M. A. Foster, A. C. Turner-Foster, A. L. Gaeta, and M. Lipson, Nat. Photonics 4, 37 (2010).
2. L. Razzari, D. Duchesne, M. Ferrera, R. Morandotti, S. Chu, B. E. Little, and D. J. Moss, Nat. Photonics 4, 41 (2010).

3. A. Liu, R. Jones, L. Liao, D. Samara-Rubio, D. Rubin, O. Cohen, R. Nicolaescu, and M. Paniccia, Nature 427, 615 (2004).

4. R. A. Myers, N. Mukherjee, and S. R. J. Brueck, Opt. Lett. 16, 1732 (1991).

5. T. Kim, J. Luo, J. Ka, S. Hau, Y. Tian, Z. Shi, N. M. Tucker, S. Jang, J. Kang, and A. K. Y. Jen, Adv. Mater. 18, 3038 (2006).

6. M. Cazzanelli, F. Bianco, E. Borga, G. Pucker, M. Ghulinyan, E. Degoli, and L. Pavesi, Nat. Mater. 11, 148 (2012).

7. M. Zdanowicz, J. Harra, J. M. Mäkelä, E. Heinonen, T. Ning, M. Kauranen, and G. Genty, Scientific Reports 4, 5745 (2014).

8. I. R. Girling, P. V. Kolinsky, N. A. Cade, J. D. Earls, and I. R. Peterson, Opt. Commun. 55, 289 (1985).

9. T. Ning, H. Pietarinen, O. Hyvärinen, J. Simonen, G. Genty, and M. Kauranen, Appl. Phys. Lett. 100, 161902 (2012).

10. A. Kitao, K. Imakita, I. Kawamura, and M. Fujii, J. Phys. D Appl. Phys. 47, 215101 (2014).

11. L. Alloatti, C. Kieninger, A. Froelich, M. Lauermann, T. Frenzel, K. Köhnle, W. Freude, J. Leuthold, M. Wegener, and C. Koos, Appl. Phys. Lett. 107, 121903 (2015).

12. A. Khanna, A. Z. Subramanian, M. Häyrinen, S. Selvaraja, P. Verheyen, D. Van Thourhout, S. Honkanen, H. Lipsanen, and R. Baets, Opt. Express 22, 5684 (2014).

13. E. Ghahramani, D. J. Moss, and J. E. Sipe, Phys. Rev. B 43, 8990 (1991).

14. N. Bloembergen, R. K. Chang, S. S. Jha, and C. H. Lee, Phys. Rev. Lett. 174, 813 (1968).

15. G. X. Liu, F. K. Shan, W. J. Lee, and B. C. Shin, J. Korean Phys Soc. 50, 1827 (2007).

16. W. Shimizu, S. Nakamura, T. Sato, and Y. Murakami, Langmuir 28, 12245 (2012).

17. J. Koo, S. Kim, S. Jeon, H. Jeon, Y. Kim, and Y. Won, J. Korean Phys. Soc. 48, 131 (2006)

18. S. K. Kim, S. W. Lee, C. S. Hwang, Y. S. Min, J. Y. Won, and J. Jeong, J. Electrochem. Soc. 153, F69 (2006).

19. D .Beena, K. J. Lethy, R. Vinodkumar, A. P. Detty, V. P. Mahadevan Pillai, and V. Ganesan, J. Alloy. Comp. 489, 215 (2011).

20. Q. Xie, Y. L. Jiang, C. Detavernier, D. Deduytsche, R. L. Van Meirhaeghe, G. P. Ru, B. Z. Li, and X. P. Qu, J. Appl. Phys. 102, 083521 (2007).

21. Q. Xie, J. Musschoot, D. Deduytsche, R. L. Van Meirhaeghe, C. Detavernier, S. Van den Berghe, Y. L. Jiang, G. P. Ru, B. Z. Li, and X. P. Qu, J. Electrochem. Soc. 155, H688 (2008).

22. R. K. Ramachandran, J. Dendooven, H. Poelman, and C. Detavernier, J. Phys. Chem. C 119, 11786 (2015).

23. R. L. Puurunen, J. Appl. Phys. 97, 121301 (2005).

24. P. Lalanne, and M. Hutley, Encyclopedia of optical engineering, 62 (2003).

25. P. D. Maker, R. W. Terhune, M. Nisenhoff, and C. M. Savage, Phys. Rev. Lett. 8, 21 (1962).

26. J. J. Maki, M. Kauranen, and A. Persoons, Phys. Rev. B 51, 1425 (1995).

27. F. J. Rodriguez, F. X. Wang, and M. Kauranen, Opt. Express 6, 8704 (2008). 


\section{References}

1. J. S. Levy, A. Gondarenko, M. A. Foster, A. C. Turner-Foster, A. L. Gaeta, and $\mathrm{M}$. Lipson, "CMOS-compatible multiple-wavelength oscillator for on-chip optical interconnects", Nat. Photonics 4, 37 (2010).

2. L. Razzari, D. Duchesne, M. Ferrera, R. Morandotti, S. Chu, B. E. Little, and D. J. Moss, "CMOS-compatible integrated optical hyper-parametric oscillator", Nat. Photonics 4, 41 (2010).

3. A. Liu, R. Jones, L. Liao, D. Samara-Rubio, D. Rubin, O. Cohen, R. Nicolaescu, and M. Paniccia, "A high-speed silicon optical modulator based on a metal-oxide-semiconductor capacitor", Nature 427, 615 (2004).

4. R. A. Myers, N. Mukherjee, and S. R. J. Brueck, "Large second-order nonlinearity in poled fused silica", Opt. Lett. 16, 1732 (1991).

5. T. Kim, J. Luo, J. Ka, S. Hau, Y. Tian, Z. Shi, N. M. Tucker, S. Jang, J. Kang, and A. K. Y. Jen, "Ultralarge and Thermally Stable Electro-optic Activities from Diels-Alder Crosslinkable Polymers Containing Binary Chromophore Systems", Adv. Mater. 18, 3038 (2006).

6. M. Cazzanelli, F. Bianco, E. Borga, G. Pucker, M. Ghulinyan, E. Degoli, and L. Pavesi, "Second-harmonic generation in silicon waveguides strained by silicon nitride", Nat. Mater. 11, 148 (2012).

7. M. Zdanowicz, J. Harra, J. M. Mäkelä, E. Heinonen, T. Ning, M. Kauranen, and G. Genty, "Second-harmonic response of multilayer nanocomposites of silver-decorated nanoparticles and silica", Scientific Reports 4, 5745 (2014).

8. I. R. Girling, P. V. Kolinsky, N. A. Cade, J. D. Earls, and I. R. Peterson, "Second harmonic generation from alternating Langmuir-Blodgett films", Opt. Commun. 55, 289 (1985).

9. T. Ning, H. Pietarinen, O. Hyvärinen, J. Simonen, G. Genty, and M. Kauranen, "Strong second harmonic generation in silicon nitride films", Appl. Phys. Lett. 100, 161902 (2012).

10. A. Kitao, K. Imakita, I. Kawamura, and M. Fujii, "An investigation into second harmonic generation by Si-rich SiNx thin films deposited by RF sputtering over a wide range of Si concentrations", J. Phys. D Appl. Phys. 47, 215101 (2014).

11. L. Alloatti, C. Kieninger, A. Froelich, M. Lauermann, T. Frenzel, K. Köhnle, W. Freude, J. Leuthold, M. Wegener, and C. Koos, "Second-order nonlinear optical metamaterials: ABC-type nanolaminates", Appl. Phys. Lett. 107, 121903 (2015).

12. A. Khanna, A. Z. Subramanian, M. Häyrinen, S. Selvaraja, P. Verheyen, D. Van Thourhout, S. Honkanen, H. Lipsanen, and R. Baets, "Impact of ALD grown passivation layers on silicon nitride based integrated optic devices for very-near-infrared wavelengths", Opt. Express 22, 5684 (2014).

13. E. Ghahramani, D. J. Moss, and J. E. Sipe, "Full-band-structure calculation of second-harmonic generation in odd-period strained $(\mathrm{Si})_{n} /(\mathrm{Ge})_{n}$ superlattices", Phys. Rev. B 43, 8990 (1991).

14. N. Bloembergen, R. K. Chang, S. S. Jha, and C. H. Lee, "Optical second Harmonic Generation in Refiection from Media with Inversion Symmetry", Phys. Rev. Lett. 174, 813 (1968).

15. G. X. Liu, F. K. Shan, W. J. Lee, and B. C. Shin, "Growth Temperature Dependence of $\mathrm{TiO}_{2}$ Thin Films Prepared by Using Plasma-Enhanced Atomic Layer Deposition Method", J. Korean Phys Soc.. 50, 1827 (2007).

16. W. Shimizu, S. Nakamura, T. Sato, and Y. Murakami, "Creation of HighRefractive-Index Amorphous Titanium Oxide Thin Films from Low-FractalDimension Polymeric Precursors Synthesized by a Sol-Gel Technique with a Hydrazine Monohydrochloride Catalyst", Langmuir 28, 12245 (2012).

17. J. Koo, S. Kim, S. Jeon, H. Jeon, Y, Kim, and Y. Won, "Characteristics of $\mathrm{Al}_{2} \mathrm{O}_{3}$ Thin Films Deposited Using Dimethylaluminum Isopropoxide and Trimethylaluminum Precursors by the Plasma-Enhanced Atomic-Layer Deposition Method", J. Korean Phys. Soc. 48, 131 (2006).

18. S. K. Kim, S. W. Lee, C. S. Hwang, Y. S. Min, J. Y. Won, and J. Jeong, "Low Temperature $\left(<100^{\circ} \mathrm{C}\right)$ Deposition of Aluminum Oxide Thin Films by ALD with $\mathrm{O}_{3}$ as Oxidant", J. Electrochem. Soc. 153, F69 (2006).

19. D .Beena, K. J. Lethy, R. Vinodkumar, A. P. Detty, V. P. Mahadevan Pillai, and V. Ganesan, "Photoluminescence in laser ablated nanostructured indium oxide thin films", J. Alloy. Comp. 489, 215 (2011).
20. Q. Xie, Y.L. Jiang, C. Detavernier, D. Deduytsche, R.L. Van Meirhaeghe, G.P. Ru, B.Z. Li, and X.P. Qu, "Atomic layer deposition of $\mathrm{TiO}_{2}$ from tetrakis-dimethyl-amido titanium or $\mathrm{Ti}$ isopropoxide precursors and $\mathrm{H}_{2} \mathrm{O}$ ", J. Appl. Phys. 102, 083521 (2007).

21. Q. Xie, J. Musschoot, D. Deduytsche, R. L. Van Meirhaeghe, C. Detavernier, S. Van den Berghe, Y. L. Jiang, G. P. Ru, B. Z. Li, and X. P. Qu, "Growth Kinetics and Crystallization Behavior of $\mathrm{TiO}_{2}$ Films Prepared by Plasma Enhanced Atomic Layer Deposition", J. Electrochem. Soc. 155, H688 (2008).

22. R. K. Ramachandran, J. Dendooven, H. Poelman, and C. Detavernier, "Low Temperature Atomic Layer Deposition of Crystalline In2O3 Films", J. Phys. Chem. C 119, 11786 (2015).

23. R. L. Puurunen, "Surface chemistry of atomic layer deposition: A case study for the trimethylaluminum/water process", J. Appl. Phys. 97, 121301 (2005).

24. P. Lalanne, and M. Hutley, "The optical properties of artificial media structured at a subwavelength scale", Encyclopedia of optical engineering, 62 (2003).

25. P. D. Maker, R. W. Terhune, M. Nisenhoff, and C. M. Savage, "Effects of Dispersion and Focusing on the Production of Optical Harmonics", Phys. Rev. Lett. 8, 21 (1962).

26. J. J. Maki, M. Kauranen, and A. Persoons, "Surface second-harmonic generation from chiral materials", Phys. Rev. B 51, 1425 (1995).

27. F. J. Rodriguez, F. X. Wang, and M. Kauranen, "Calibration of the secondorder nonlinear optical susceptibility of surface and bulk of glass", Opt. Express 6, 8704 (2008). 